\title{
RESEARCH AND PRACTICE IN ANESTHESIOLOGY
}

\section{Research}

\section{"Corresponding author}

Ong Ee Teng, MBBS, MMed Anathesia

Department of Anaesthesia

Singapore General Hospital

Block 5 Level 2

Outram Road

Singapore 169608

E-mail: eeteng.ong@gmail.com

Volume 2 : Issue 1

Article Ref. \#: 1000AOJ2108

\section{Article History}

Received: January $8^{\text {th }}, 2017$

Accepted: February $13^{\text {th }}, 2017$

Published: February $13^{\text {th }}, 2017$

\section{Citation}

Ong ET, Ng SY, Yang Y, Thong SY Factors affecting post-operative mortality rate after major lower extremity amputations in a tertiary institution in Singapore. Res Pract Anesthesiol Open J. 2017; 2(1): 1-8. doi: 10.17140/RPAOJ-2-108

\section{Copyright}

(C)2017 Teng OE. This is an open access article distributed under the Creative Commons Attribution 4.0 International License (CC BY 4.0), which permits unrestricted use, distribution, and reproduction in any medium, provided the original work is properly cited.

\section{Factors Affecting Post-Operative Mortality Rate after Major Lower Extremity Amputations in a Tertiary Institution in Singapore}

Ong Ee Teng, MBBS, MMed Anathesia*; Ng Shin Yi, MMed Anaesthesia, EDIC, FCCP, FAMS; Yang Yong, MBBS, MMed, MSc, PhD; Thong Sze Ying, MBBS, MMed Anaesthesia, FANZCA

Department of Anaesthesia, Singapore General Hospital, Block 5 Level 2, Outram Road, Singapore 169608

\section{ABSTRACT}

Background: Non-traumatic Major Lower Extremity Amputation (MLEA) surgeries are associated with high post-operative morbidity and mortality rates. There are few studies evaluating factors associated with post-operative mortality rates for MLEA among Asian patients.

Objective: To identify factors that affect post-operative mortality rate and ICU admission rates in patients undergoing non-traumatic MLEA surgeries in a tertiary institution in Singapore.

Method: A retrospective study was performed by using the operating theatre electronic database to identify patients who underwent non-traumatic MLEA surgeries in Singapore General Hospital during the period of January 1, 2010 to December 31, 2011.

Results: Data was collected from 186 non-traumatic MLEA surgeries performed during the study period. Overall post-operative in hospital mortality rate was $8.6 \%$, higher for above knee amputation (AKA), than Through Knee Amputation (TKA) than Below Knee Amputation (BKA) $(18.4 \%$ vs. $8 \%$ vs. $4.5 \%, p=0.015)$. In multivariate logistic regression analysis, site of amputation of AKA compared to BKA (odds ratio 3.9, 95\% confidence interval 1.1-14.5, $p=0.04$ ), ASA 4 status (odds ratio 4.3, 95\% confidence interval 1.2-14.6, $p=0.02$ ) and presence of septic shock (odds ratio $4.9,95 \%$ confidence interval $1.4-17.3, p=0.01$ ) were significant predictors of post-operative in hospital mortality rate. The same 3 factors were significant predictors of post-operative ICU admission rate. Use of peripheral nerve block as the sole anaesthetic technique did not affect in-hospital mortality rate (adjusted odds ratio 1.3, 95\% confidence interval 0.3-5.6).

Conclusion: Patients with ASA 4 status and pre-operative septic shock, especially those undergoing AKA, should be considered as high risk group and therefore managed appropriately. The higher incidence of post-operative ICU admission rate in this group of patients also necessitates pre-operative organization of bed availability. Using peripheral nerve block as the sole anaesthetic technique did not significantly affect outcomes measured.

KEY WORDS: Major lower extremity amputation; In-hospital mortality rate; Peripheral nerve block.

ABBREVIATIONS: MLEA: Major Lower Extremity Amputation; IRB: Institutional Review Board; BKA: Below Knee Amputation; TKA: Through Knee Amputation; AKA: Above Knee Amputation.

\section{INTRODUCTION}

Non-traumatic major lower extremity amputation (MLEA) surgeries are associated with significant post-operative morbidity and mortality. Studies have found the 30-day mortality rate for this group of patients to be ranging from $7-23 \% .^{1-7}$ Patients undergoing these operations 


\section{RESEARCH AND PRACTICE IN ANESTHESIOLOGY}

often have significant multiple comorbidities ${ }^{4}$ and pre-operative hemodynamic disturbances, putting them at high risk both for surgery and for the further hemodynamic changes associated with anaesthesia. Most studies evaluating factors associated with peri-operative mortality rates in this group of patients are conducted in Caucasian populations, ${ }^{3-6}$ and we find a lack of similar studies evaluating these factors in the Asian population.

We performed a retrospective review of all MLEA surgeries that took place in Singapore General Hospital across a two year period to evaluate the factors that affect post-operative morbidity and mortality rate. Post-operative in hospital mortality rate, intensive care unit (ICU) admission rate and incidence of medical and surgical complications were assessed.

In this study, we also did a subgroup analysis to evaluate if using peripheral nerve block as the sole intra-operative anaesthetic technique affects in-patient mortality and ICU admission rates.

\section{DESIGN AND METHODS}

We obtained approval from the Singhealth Centralised Institutional Review Board (IRB) to conduct this study. A review of the operating theatre electronic database was done to identify patients who underwent MLEA surgeries, including below knee amputation (BKA), through knee amputation (TKA) and above knee amputation (AKA), for the period of January 1, 2010 to December 31, 2011, in Singapore General Hospital.

We manually traced the intra-operative anaesthesia chart and paper inpatient records for all 206 surgeries identified. A review of the electronic hospital records for these 206 surgeries was also done if the paper records were insufficient to provide us with the data required.

Data collection was done by only 2 investigators to ensure fidelity of data collection. Data that was not available on the paper anaesthesia chart and inpatient notes were then supplemented by the electronic hospital records. As such, there was no missing data.

Data was collected for demographic variables, preoperative comorbidities, date and type of surgical procedure including repeat operations, post-operative ICU admission and occurrence of complications including in-patient mortality, medical and surgical complications.

Demographic variables collected include, age, gender and race. Pre-operative comorbidities looked at include diabetes mellitus, and whether patient was insulin-dependent, hypertension, ischaemic heart disease and chronic renal impairment, including whether patient was dialysis dependent. We defined chronic renal impairment as serum creatinine $>2 \mathrm{mg} / \mathrm{dL}$ or $>176 \mathrm{umol} / \mathrm{L}$. Patient's assigned ASA status was noted from the pre-operative assessment on the anaesthesia chart. Presence of medical contraindications to central neuraxial block was recorded, including presence of coagulopathy with INR $>1.2$, use of anti-platelet agents which contraindicate the use of neuraxial anaesthesia and anti-coagulants. Presence of septic shock was identified, and defined as sepsis in the presence of pre-induction hypotension with mean arterial pressure $<65 \mathrm{mmHg}$ for 2 consecutive readings, and/or pre-operative or intra-operative requirement for vasoactive drugs, including one of more of adrenaline, noradrenaline, dopamine and/or dobutamine. This is based on the 2001 definition of septic shock.

The anaesthesia chart was reviewed for choice of anaesthesia technique between general anaesthesia, central neuraxial block or peripheral nerve block. If peripheral nerve block was the technique of choice, the success of the block was noted, including whether supplementation with analgesia was required, or whether the surgery necessitated conversion to general anaesthesia.

Post-operative admission into ICU was noted, including days of ICU admission. Incidence of post-operative medical complications collected include: pneumonia, acute myocardial infarction, cerebrovascular accident, urinary tract infection or new onset arrhythmias. Incidence of post-operative surgical complications collected include presence of sepsis, wound infection, pressure sore, bleeding requiring blood transfusion and repeat operations. Repeat operations could include a higher level amputation or wound debridement of the existing amputation site.

\section{STATISTICAL ANALYSES}

Continuous outcomes are given as the mean $\pm \mathrm{SD}$ and categorical data are presented as percentages. Univariate analysis was used to identify individual variables that could be associated with inpatient mortality and ICU admission. A less stringent $p$-value of 0.10 was used during univariate analysis so as to include all variables that could affect outcomes into the final analysis. These factors were then entered into a multivariate logistic regression analysis to identify independent variables that affect the outcomes of mortality rate and ICU admission rate. A final $p$ value $<0.05$ was considered statistically significant. All statistical analyses were conducted using the Statistical Package for the Social Sciences (SPSS, version 17.0).

In the subgroup analysis, we divided patients into those who received peripheral nerve block for their surgeries (PNB group) and those who did not receive peripheral nerve block for their surgeries (non-PNB group). PNB group of patients was defined as those who received only peripheral nerve block for their surgeries, including femoral nerve block together with sciatic or popliteal nerve block, and/or obturator nerve block, depending on the level of amputation. The non-PNB group was defined as those patients receiving either general anaesthesia or central neuraxial block for their surgeries. For patients who underwent peripheral nerve block, the anaesthesia chart was reviewed for 


\section{RESEARCH AND PRACTICE IN ANESTHESIOLOGY

success of the block. Failure of peripheral nerve block was defined as insertion of an airway device with continuous administration of intravenous or inhalational anaesthetic agent at any point during the surgery. Patients who had complete failure of peripheral nerve block necessitating conversion to general anaesthesia, with insertion of an airway device, were analysed under the non-PNB group.

\section{RESULTS}

Within the study period, there were 206 MLEA surgeries performed in 186 patients. Four patients who had lower limb amputations due to trauma and 1 patient due to tumour were excluded from the study as they are recognised to have a different disease process and baseline characteristics from patients who underwent surgery due to ischaemia or infection. 11 patients had 2 amputation surgeries and 2 patients had 3 amputation surgeries within the study period. For these patients with repeated lower limb amputations, only the first operation was included in the data analysis.

After exclusion of the repeat surgeries and patients who had surgery due to tumour or trauma, a total of 186 surger- ies, including 112 BKA (60.2\%), 25 TKA (13.4\%) and 49 AKA $(26.3 \%)$ were included in the data analysis. The study flow chart is shown in Figure 1.

Table 1 shows the baseline characteristics of the patients divided into the 2 subgroups of peripheral nerve block versus no peripheral nerve block. Baseline characteristics of patients in both groups are comparable. However, patients in the PNB group were 2.7 times more likely to have ischaemic heart disease (confidence interval 1.1-6.7) and 4 times more likely to have medical contraindications to central neuraxial block (confidence interval 1.6-9.5).

Overall post-operative in-hospital mortality rate was $8.6 \%$ (16/186 operations). The in-hospital mortality rate was worst for AKA (18.4\%), than TKA (8\%) and BKA (4.5\%) patients $(p=0.015)$. Post-operative day of death ranged from POD 3 to POD 83.

On univariate analysis, variables that predicted in hospital mortality with $p<0.10$ were DM $(p=0.02)$, presence of septic shock $(p=0.014)$, site of amputation $(p=0.015)$ and ASA status $(p=0.027)$. These variables, as well as age group and the

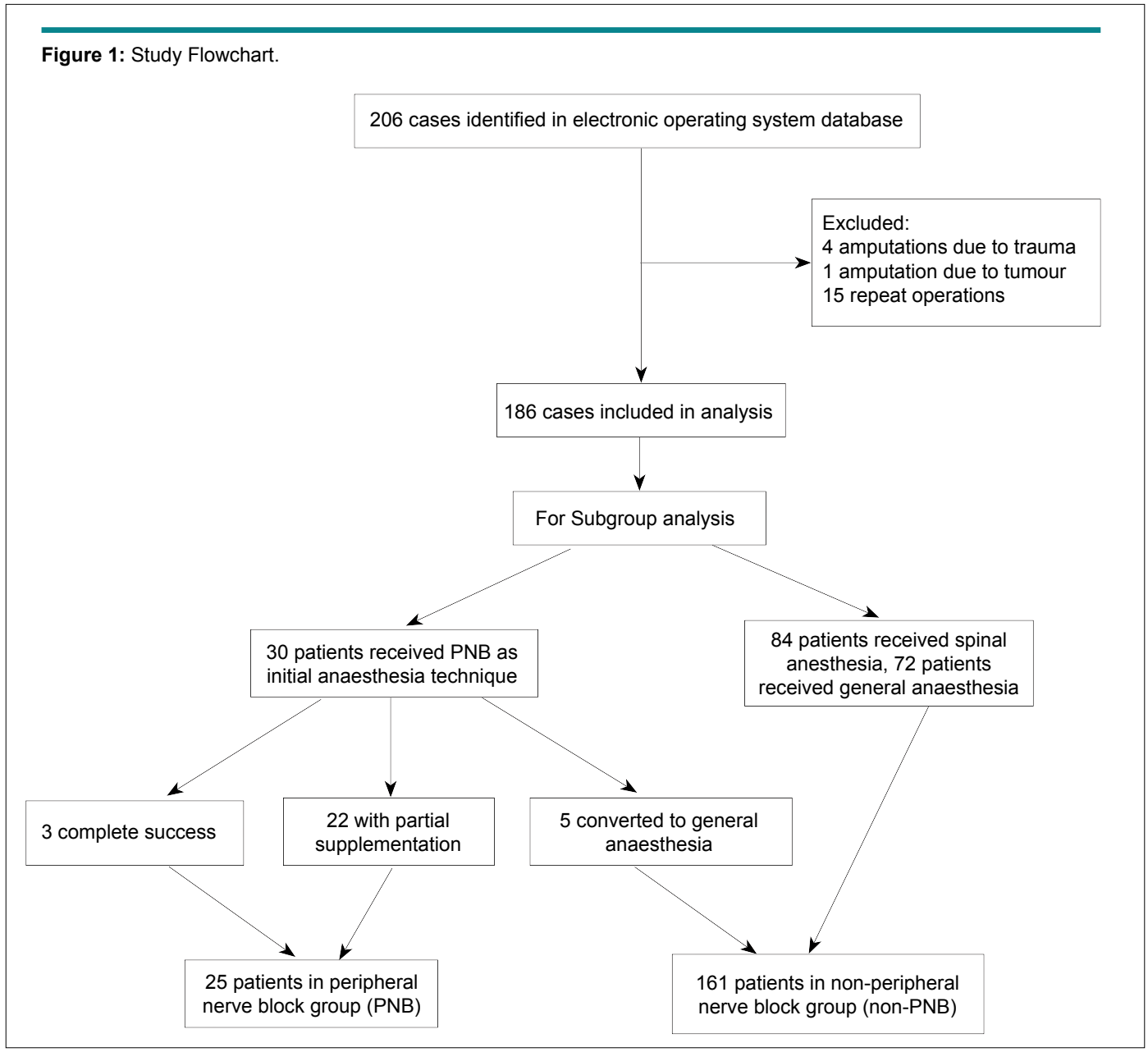




\section{RESEARCH AND PRACTICE IN ANESTHESIOLOGY}

\begin{tabular}{|c|c|c|c|}
\hline & Non-PNB group (total 161) & PNB group (total 25) & p-Value \\
\hline \multicolumn{4}{|l|}{ Age } \\
\hline$<65$ & $80(50 \%)$ & $12(48 \%)$ & \multirow{2}{*}{0.878} \\
\hline$>65$ & $81(50 \%)$ & $13(52 \%)$ & \\
\hline \multicolumn{4}{|l|}{ Gender } \\
\hline Male & $95(59 \%)$ & $151(60 \%)$ & \multirow[t]{2}{*}{0.93} \\
\hline Female & $66(41 \%)$ & $10(40 \%)$ & \\
\hline \multicolumn{4}{|l|}{ Race } \\
\hline Chinese & $105(65 \%)$ & $15(60 \%)$ & \multirow[t]{4}{*}{0.59} \\
\hline Malay & $31(19 \%)$ & $7(28 \%)$ & \\
\hline Indian & $22(14 \%)$ & $2(8 \%)$ & \\
\hline Others & $3(2 \%)$ & $1(4 \%)$ & \\
\hline \multicolumn{4}{|c|}{ Diabetes Mellitus } \\
\hline No & $14(9 \%)$ & $3(12 \%)$ & \multirow[t]{2}{*}{0.59} \\
\hline Yes & $147(91 \%)$ & $22(88 \%)$ & \\
\hline \multicolumn{4}{|l|}{ Hypertension } \\
\hline No & $27(117)$ & & \multirow[t]{2}{*}{0.55} \\
\hline Yes & $134(83 \%)$ & $22(88 \%)$ & \\
\hline \multicolumn{4}{|c|}{ I schaemic Heart disease } \\
\hline No & $79(49 \%)$ & $1(20 \%)$ & \multirow[t]{2}{*}{0.03} \\
\hline Yes & $79(49 \%)$ & $18(/ 2 \%)$ & \\
\hline \multicolumn{4}{|c|}{ Chronic renal impairment } \\
\hline No & $00(51 \%)$ & $12(40 \%)$ & \multirow{2}{*}{0.74} \\
\hline Yes & $78(48 \%)$ & $13(52 \%)$ & \\
\hline \multicolumn{4}{|c|}{ Pre-operative septic shock } \\
\hline No & $92(57 \%)$ & $10(40 \%)$ & \multirow[t]{2}{*}{0.11} \\
\hline Yes & $69(43 \%)$ & $15(60 \%)$ & \\
\hline \multicolumn{4}{|l|}{ ASA } \\
\hline 3 & $113(70 \%)$ & $14(56 \%)$ & \multirow[t]{2}{*}{0.16} \\
\hline 4 & $48(30 \%)$ & $11(44 \%)$ & \\
\hline \multicolumn{4}{|c|}{$\begin{array}{l}\text { Medical contraindication to central neuraxial } \\
\text { block }\end{array}$} \\
\hline No & $111(68 \%)$ & $9(36 \%)$ & \multirow{2}{*}{0.001} \\
\hline Yes & $50(31 \%)$ & $16(64 \%)$ & \\
\hline \multicolumn{4}{|c|}{ Site of amputation } \\
\hline BKA & $97(60 \%)$ & $15(60 \%)$ & \multirow{3}{*}{0.90} \\
\hline TKA & $21(13 \%)$ & $4(16 \%)$ & \\
\hline AKA & $43(27 \%)$ & $6(24 \%)$ & \\
\hline
\end{tabular}

use of peripheral nerve block as the sole anaesthetic technique were entered into a multivariate logistic regression analysis of post-operative inpatient mortality rate (Table 2).

AKA site of operation, presence of ASA 4 status, and septic shock were significant predictors of post-operative in hospital mortality rate. The use of peripheral nerve block as the sole intra-operative anaesthetic technique did not significantly affect post-operative in hospital mortality rate $(p=0.77)$.

Overall rate of ICU admission was 7.5\%, worst for AKA $(14.3 \%)$ than TKA ( $8 \%)$ and BKA $(4.5 \%)$ patients $(p=0.09)$. On univariate analysis, variables that predicted post-operative ICU admission with $p<0.10$ was septic shock $(p=0.002)$, the use of peripheral nerve block as the sole anaesthesia technique $(p=0.084)$, site of amputation $(p=0.094)$ and ASA status $(p=0.001)$. These variables, as well as age group and presence of diabetes mellitus were entered into a multivariate logistic regression analysis for incidence of post-operative ICU admission. Again, site of amputation of AKA compared to BKA, ASA 4 status and presence of septic shock were predictors of post-operative ICU admission. (Table 3). Patients with septic shock were 15 times more likely to require post-operative ICU admission, AKA patients were 5.5 times more likely that BKA patients to require post-operative ICU admission and ASA 4 patients were 8.6 times more likely to be admitted to ICU post operatively. 


\section{RESEARCH AND PRACTICE IN ANESTHESIOLOGY}

\begin{tabular}{|c|c|c|}
\hline Variable & $\begin{array}{c}\text { Adjusted Odds ratio } \\
\text { (95\% confidence interval) }\end{array}$ & $p$-Value \\
\hline \multicolumn{3}{|l|}{ Age Group } \\
\hline$<65$ & Reference & \\
\hline$>65$ & $1.5(0.4-5.3)$ & 0.57 \\
\hline \multicolumn{3}{|l|}{ Site of operation } \\
\hline BKA & Reference & \\
\hline TKA & $0.8(0.1-5.2)$ & 0.77 \\
\hline AKA & $3.9(1.1-14.5)$ & 0.04 \\
\hline \multicolumn{3}{|l|}{ ASA Group } \\
\hline ASA 3 & Reference & \\
\hline ASA 4 & $4.3(1.2-14.6)$ & 0.02 \\
\hline Use of peripheral nerve block & $1.3(0.3-5.6)$ & 0.77 \\
\hline Presence of septic shock & $4.9(1.4-17.3)$ & 0.01 \\
\hline Diabetes Mellitus & $0.2(0.1-1.1)$ & 0.07 \\
\hline
\end{tabular}

\begin{tabular}{|c|c|c|}
\hline Variable & $\begin{array}{c}\text { Adjusted odds ratio } \\
\text { (95\% confidence interval) }\end{array}$ & $p$-value \\
\hline \multicolumn{3}{|l|}{ Age group } \\
\hline$<65$ & Reference & \\
\hline$>65$ & $0.7(0.2-2.8)$ & 0.59 \\
\hline \multicolumn{3}{|l|}{ Site of amputation } \\
\hline BKA & Reference & \\
\hline TKA & $0.7(0.1-5.4)$ & 0.72 \\
\hline AKA & $5.5(1.2-25.5)$ & 0.03 \\
\hline \multicolumn{3}{|l|}{ ASA group } \\
\hline ASA 3 & Reference & \\
\hline ASA 4 & $8.6(2.1-35.4)$ & 0.003 \\
\hline Use of peripheral nerve block & $3.5(0.8-15.4)$ & 0.11 \\
\hline Presence of septic shock & $15.2(2.5-94.5)$ & 0.003 \\
\hline Diabetes Mellitus & $0.8(0.1-6.0)$ & 0.81 \\
\hline
\end{tabular}

Across all 3 types of MLEA surgeries, incidence of surgical complications was $33.3 \%$ and incidence of medical complications was $22.6 \%$ (Table 4 ).

The 30 patients who received peripheral nerve block as the sole anaesthesia technique, 3 patients had complete success of the nerve block, 22 patients required partial supplementation of the nerve block and 5 nerve blocks were complete failure and required conversion to general anaesthesia with insertion of an airway device. The failure rate of PNB was $16.7 \%$. 22 patients had their nerve block supplemented with intravenous analgesic or sedative agents, including fentanyl, propofol and/or ketamine, without the need for insertion of airway device or conversion to general anaesthesia.

In-hospital mortality rate was $12 \%$ for the PNB group and $8 \%$ for the non-PNB group of patients but the difference was not statistically significant via univariate analysis (Table 5). After adjustment for age, presence of diabetes, septic shock, ASA status, site of amputation, presence of ischaemic heart disease and presence of medical contraindication to central neuraxial block via logistic regression analyses, the use of peripheral nerve block was not independently associated with in hospital mortality rate (adjusted odds ratio $1.25,95 \%$ confidence interval $0.3-5.6, p=0.77)$.

Post-operative ICU admission rate was $16 \%$ for the PNB group and $6 \%$ for the non-PNB group but the difference was again not statistically significant via univariate analysis. There was no significant association between use of peripheral nerve block and incidence of post-operative ICU admission rate (adjusted odds ratio 3.5, 95\% confidence interval 0.8-15.4, 


\section{RESEARCH AND PRACTICE IN ANESTHESIOLOGY}

\begin{tabular}{|c|c|c|}
\hline Complications & Number of patients & Percentage (\%) \\
\hline \multicolumn{3}{|l|}{ Surgical complications } \\
\hline Sepsis & 31 & 16.7 \\
\hline Wound infection & 22 & 11.8 \\
\hline Repeat operation & 16 & 8.6 \\
\hline Bleeding & 15 & 8.1 \\
\hline Pressure sore & 3 & 1.6 \\
\hline Total surgical complications & 62 & 33.3 \\
\hline \multicolumn{3}{|l|}{ Medical complications } \\
\hline Acute myocardial infarction & 18 & 9.7 \\
\hline Pneumonia & 16 & 8.6 \\
\hline Urinary Tract Infection & 13 & 7.0 \\
\hline Cerebrovascular Accident & 4 & 2.2 \\
\hline Arrhythmias & 4 & 2.2 \\
\hline Total Medical complications & 42 & 22.6 \\
\hline
\end{tabular}

\begin{tabular}{|c|c|c|}
\hline & No PNB used & PNB used \\
\hline Alive & 148 & 22 \\
\hline In-hospital mortality & 13 & 3 \\
\hline Total & 161 & 25 \\
\hline
\end{tabular}

$p=0.105)$

\section{DISCUSSION}

Major lower extremity amputation (MLEA) surgeries place a huge burden on the healthcare system due to its high associated post-operative morbidity and mortality rates. ${ }^{1-7}$ With the increasing incidence of diabetes mellitus in Asia, the incidence of MLEA surgeries is set to increase over the next few years ${ }^{8-10}$ as diabetes is one of the major risk factors for critical limb ischaemia and lower limb amputation surgeries. ${ }^{10}$ It is important to identify factors that affects outcome in this group of patients.

In our study, we analysed outcomes of a predominantly Asian population undergoing MLEA to identify the factors that significantly predict post-operative in hospital mortality and ICU admission rates. With the increasing interest in regional anaesthesia, we also tried to investigate if using peripheral nerve block as the sole anaesthetic technique can influence these outcomes.

Our study found that local outcome after non-traumatic MLEA was similar to that found in other studies and population groups. Post-operative in hospital mortality rate for non-traumatic MLEA was $8.6 \%$ in our centre, which is consistent with earlier studies that found a 30 -day mortality rate of $7-23 \%{ }^{1-7} \mathrm{~A}$ significantly higher mortality rate for AKA (18.4\%) than BKA patients $(4.5 \%)$ is also consistent with previous studies. ${ }^{2}$

There are several significant findings in our study. Firstly, pre-operative septic shock, ASA 4 status and site of amputation of AKA are significant predictors of higher post-operative in hospital mortality rate and ICU admission. Previous studies evaluating non-traumatic MLEA found thatage, ${ }^{6}$ albumin level, ${ }^{6}$ AKA,${ }^{4,6}$ lack of a previous coronary artery bypass graft, ${ }^{6}$ renal insufficiency, ${ }^{4}$ ASA grade $\geq 4^{12}$ and out-of-hours surgery ${ }^{12}$ are factors that affect peri-operative mortality rate. This knowledge is important when risk stratifying patients before surgery. Appropriate post-operative disposition can also be arranged before the operation.

Secondly, we found that using peripheral nerve block as the sole intra-operative anaesthetic technique did not lower incidence of post-operative mortality and ICU admission rates. However, with the small number of patients in the PNB group and the differences in baseline characteristics between the PNB and non-PNB group of patients, we must interpret the results with caution. Although logistic regression analysis was used to adjust for known confounders identified in our study, we were not able to account for unknown confounders that may be present. Further research with randomised controlled trials would be necessary to make meaningful conslusions. Although peripheral 


\section{RESEARCH AND PRACTICE IN ANESTHESIOLOGY}

nerve block has been shown to provide greater intra-operative hemodynamic stability when used as the sole anaesthetictechnique, ${ }^{13,14}$ there is a lack of studies to prove that this translates to improved post-operative outcomes, including a lowered postoperative mortality rate. ${ }^{14}$

There is mixed evidence in the literature when evaluating whether anaesthesia technique affects mortality rate in MLEA surgeries. One study found that the type of anaesthetic (neuraxialvs general) was not associated with survival when corrected for other variables, ${ }^{12}$ while other studies show that avoiding general anaesthesia in favour of regional anaesthesia may improve post-operative outcomes in non-traumatic MLEA surgeries. ${ }^{7,16}$ However, all these studies did not distinguish between central neuraxial block and peripheral nerve block in the group analysed under regional anaesthesia. One large scale retrospective study showed that 30-day mortality is significantly higher in patients undergoing MLEA under general anaesthesia compared with regional anaesthesia with an odds ratio of $1.5 .^{7}$ However when further subgroup analyses was done for patients that underwent knee amputation surgery under peripheral nerve block alone, the authors was unable to detect any significant difference of the effect of anaesthesia on 30-day mortality rate.

While both peripheral nerve block and central neuraxial block avoid the risks associated with airway instrumentation in general anaesthesia, spinal anaesthesia can significantly decrease mean arterial pressure and cardiac index even in healthy ASA 1 and 2 patients, whereas peripheral nerve block provide a more hemodynamically stable anaesthesia, ${ }^{17}$ The characteristics of patients who receive spinal anaesthesia may be different from those who receive a peripheral nerve block for a MLEA, with the former being less septic, hemodynamically stable, and with an intact coagulation profile. Patients who can receive central neuraxial block are also not on multiple antiplatelet and anticoagulant agents that contraindicate the block, which may indicate a lower incidence of underlying cardiovascular comorbidities that necessitate such drugs. Grouping these two heterogeneous groups of patients together for analysis of mortality outcomes may skew results, making readers unable to tell the true effect of using a peripheral nerve block alone.

When given the choice, most physicians may choose to perform spinal anaesthesia over peripheral nerve block for a patient undergoing MLEA, because of the increased predictability, higher success rate, faster onset and lower technical difficulty of the former. A recent census report conducted in Greece in 2015 supported this view that many anaesthesiologists may not be familiar with peripheral nerve blocks. ${ }^{16}$ In addition to the individual anaesthetist's experience and training in regional anaesthesia, consideration also needs to be given to the possible failure of PNB, time required for block performance and block onset, as well as patient preference. The aftermath of a failed block requiring supplementation and conversion to GA may be a very negative experience for everyone involved, including the anaesthetist, surgeon and patient, leading to a future aversion to
PNB in similar operations.

With all these considerations, anaesthetists will require stronger evidence that peripheral nerve block may influence outcomes in high risk surgeries such as AKA, to guide their decision making towards an appropriate anaesthetic technique choice. However, we were unable to find a significant association between the use of peripheral nerve block and post-operative in-hospital mortality and ICU admission rate in our study. In a review article, ${ }^{18}$ looking at evidence for whether regional anaesthesia improves outcome, the authors concede that it may be unrealistic to expect studies with results showing regional anaesthesia improving peri-operative mortality rate. As given the safety of modern anaesthesia with low anaesthesia-related mortality rates, we may require randomised controlled trials to include millions of patients before we can detect significant differences in mortality rate. ${ }^{18}$ Instead, it may be more fruitful for future studies to look at how peripheral nerve blocks may improve specific outcome parameters in the post-operative period.

In addition to flaws inherent to retrospective study design, our study may be underpowered to detect all factors that may influence outcomes in MLEA or rare complications after anaesthesia. Secondly, our data review collected in-patient mortality rate with no follow-up beyond that for 30-day or 1 year mortality rate. Hence, the advantages of a specific technique beyond this period cannot be detected. We used logistic regression analysis to adjust for possible confounders, including age, ASA status, diabetes mellitus, pre-operative septic shock and site of amputation. Conducting a large scale randomised controlled trial will be much more difficult, involving significant manpower, time, economical and ethical issues. Lastly, as with all database-based studies, errors may exist in the data-collection and data-entry phase. However, we tried to overcome this by using multiple sources of information for data collection, including the anaesthesia chart, electronic discharge summary records and paper inpatient notes, to corroborate patients' clinical information.

\section{CONCLUSION}

Non traumatic major lower extremity amputations, especially above knee amputations, are high risk surgeries associated with significant peri-operative mortality and morbidity rates. ${ }^{1-7}$ Patient's pre-operative condition, including their ASA status, presence of septic shock and site of amputation undertaken are significant predictors of post-operative in hospital mortality rate and ICU admission rate. Patients with pre-operative septic shock, ASA 4 status and undergoing AKA should be regarding as high risk group, and appropriate level of intra-operative monitoring and post-operative disposition should be arranged. Using peripheral nerve block as the sole anaesthetic technique was not a significant predictor of in hospital mortality rate or ICU admission rate. Further large scale studies are required to determine whether peripheral nerve blocks may influence specific outcomes in these high risk surgeries. 


\section{RESEARCH AND PRACTICE IN ANESTHESIOLOGY

\section{CONFLICTS OF INTEREST}

The authors declare that they have no conflicts of interest.

\section{FUNDING}

The author(s) received no financial support for the research, authorship, and/or publication of this article.

\section{REFERENCES}

1. Kald A, Carlsson R, Nilsoon E. Major amputation in a defined population: Incidence, mortality and results of treatment. $\mathrm{Br} J$ Surg. 1989; 76(3): 308-310. doi: 10.1002/bjs.1800760328

2. Moxey PW, Hofman R, Hinchliffe RJ. Epidemiological study of lower limb amputation in England between 2003 and 2008. Br J Surg. 2010; 97(9): 1348-1353. doi: 10.1002/bjs.7092

3. Kazmers A, Perkins AJ, Jacobs LA. Major lower extremity amputation in Veterans Affairs medical centers. Ann Vasc Surg. 2000; 14(3): 216-222. doi: 10.1007/s100169910038

4. Subramaniam B, Pompselli F, Talmor D. Peri-operative and long-term morbidity and mortality after above-knee and below-knee amputations in diabetics and non-diabetics. Anesth Analg. 2005; 100(5): 1241-1247. doi: 10.1213/01.ANE. 0000147705.94738 .31

5. Bernadette A, Hile CN, Hamdan AD, et al. Major Lower Extremity Amputation. Outcome of a modern series. Arch Surg. 2004; 139(4): 395-399. doi: 10.1001/archsurg.139.4.395

6. Stone PA, Flaherty SK, Aburahma AF, et al. Factors affecting mortality and wound-related complications following major lower extremity amputations. Ann Vasc Surg. 2006: 20(2): 209221. doi: 10.1007/s10016-006-9009-z

7. Khan SA, Qianyi RL, Liu C, Ng EL, Fook-Chong S, Tan MG. Effect of anaesthetic technique on mortality following major lower extremity amputation. Anaesthesia. 2013; 68(6): 612-620. doi: 10.1111/anae.12182

8. Wild S, Roglic G. Global prevalence of diabetes: Estimates for the year 2000 and projections for 2010. Diabetes Care. 2004; 27(10): 1047-1053. doi: 10.2337/diacare.27.10.2568
9. Yoon KH, Lee JH. Epidemic Obesity and type 2 diabetes in Aisa. Lancet. 2006; 368(9548): 1681-1688.

10. Tan ML, Feng J, Gordois A, Wong ES. Lower extremity amputation prevention in Singapore: Economic analysis of results. Singapore Med J. 2011; 52(9): 662-668. Web site. http:// apamedcentral.org/search.php?where $=$ aview \&id $=10.0000 \% 2 \mathrm{~F}$ smj.2011.52.9.662\& code $=0022$ SMJ $\&$ vmode $=$ FULL. Accessed January 7, 2017.

11. Ploeg AJ, Lardenoye JW, Vrancken Peeters MP, Breslau PJ. Contemporary series of Morbidity and Mortality after Lower limb amputation. Eur J Vasc Endovasc Surg. 2005; 29(6): 633637. doi: $10.1016 /$ j.ejvs.2005.02.014

12. Scott SW, Bowrey S, Clarke D, Choke E, Bown MJ, Thompson JP. Factors affecting short- and long-term mortality after lower limb amputation. Anaesthesia. 2014; 69(3): 249-258. doi: 10.1111/anae. 12532

13. Maximillian H. Peripheral nerve blocks give greater hemodynamic stability than general anaesthesia for ASA III patients undergoing outpatient knee arthroscopy. Cent Eur J Med. 2013; 8(4): 436-442. doi: 10.2478/s11536-012-0143-4

14. Baddoo HK. A preliminary report on the use of peripheral nerve block for lower limb amputation. Ghana Med J. 2009; 43(1): 24-28.

15. Lin R, Hingorani A. Effects of anaesthesia versus regional nerve block on major leg amputation mortality rate. Vascular. 2013; 21(2): 83-86. doi: 10.1177/1708538113478718

16. Argyra E, Moka E, Staikou C, et al. Regional anaesthesia practice in Greece: A census report. $J$ Anaesthesiolol Clin Pharmacol. 2015; 31(1): 59-66. doi: 10.4103/0970-9185.150545

17. Fanelli G, Casati A, Aldegheri G, et al. Cardiovascular effects of two different regional anaesthetic techniques for unilateral leg surgery. Acta Anaesthesiol Scand. 1998; 42(1): 80-84. doi: 10.1111/j.1399-6576.1998.tb05084.x

18. Kettner SC, Willschke H, Marhofer P. Does regional anaesthesia really improve outcome? Br J Anaesth. 2011; 107(S1): i90-i95. doi: 10.1093/bja/aev377 